\title{
The Benefits and Challenges of Setting Up a Longitudinal Psoriatic Arthritis Database
}

\author{
Dafna D. Gladman, Laura C. Coates, Deepak R. Jadon, William Tillett, Philip J. Mease, \\ and Marijn Vis
}

ABSTRACT. The members of the Group for Research and Assessment of Psoriasis and Psoriatic Arthritis (GRAPPA) have shown great interest in developing a common GRAPPA database. To address this interest, GRAPPA included a symposium at its 2017 annual meeting to examine the concepts of registries and databases. At this symposium, examples of existing databases were reviewed, and their challenges and achievements were discussed. (J Rheumatol Suppl. 2018 June;94:26-9; doi:10.3899/ jrheum.180132)

\section{Key Indexing Terms: \\ DATABASES PSORIASIS PSORIATICARTHRITIS REGISTRIES PROGNOSIS GRAPPA}

Psoriatic arthritis (PsA) is a complex condition characterized by a variety of clinical manifestations and disease courses. As with other similar conditions, the best way to understand the course of disease and patient prognosis is through observational cohort studies. These studies depend on the prospective collection of data on a large number of patients followed according to standard protocols ${ }^{1}$. At the GRAPPA 2017 annual meeting in Amsterdam, the Netherlands, examples of existing databases were reviewed and their challenges and achievements were discussed.

Dr. Dafna D. Gladman (Toronto, Ontario, Canada) discussed the differences between registries and cohorts. She stated that there are several types of registries, including

From the University of Toronto; Krembil Research Institute; Psoriatic Arthritis Program University Health Network, Toronto Western Hospital, Toronto, Ontario, Canada; Department of Orthopedics, Rheumatology, and Musculoskeletal Sciences, University of Oxford, Oxford; Department of Rheumatology, Cambridge University Hospitals National Health Service (NHS) Foundation Trust, Cambridge; Royal National Hospital for Rheumatic Diseases; University of Bath, Bath, UK; Swedish Medical Center and University of Washington School of Medicine, Seattle, Washington, USA; Department of Rheumatology, Erasmus Medical Center Rotterdam, the Netherlands.

As part of the supplement series GRAPPA 2017, this report was reviewed internally and approved by the Guest Editors for integrity, accuracy, and consistency with scientific and ethical standards.

D.D. Gladman, MD, FRCPC, Professor of Medicine, University of Toronto, and Senior Scientist, Krembil Research Institute, and Director, Psoriatic Arthritis Program University Health Network, Toronto Western Hospital; L.C. Coates, MBChB, PhD, Department of Orthopedics, Rheumatology, and Musculoskeletal Sciences, University of Oxford; D.R. Jadon, MBBCh, MRCP, PhD, Consultant Rheumatologist,

Department of Rheumatology, Cambridge University Hospitals NHS Foundation Trust; W. Tillett, MBChB, PhD, Consultant Rheumatologist, Royal National Hospital for Rheumatic Diseases, and Senior Lecturer, University of Bath; P.J. Mease, MD, Rheumatology Research, Swedish Medical Center and University of Washington School of Medicine; M. Vis, MD, Department of Rheumatology, Erasmus Medical Center, Rotterdam, the Netherlands.

Address correspondence to Dr. D.D. Gladman, Toronto Western Hospital, 399 Bathurst St., 1E-410B, Toronto, Ontario M5T 2S8, Canada.

E-maildafna.gladman@utoronto.ca administrative registries, registries for clinical trials, registries for genetic studies, registries for biologics, and registries for longitudinal observational studies.

Administrative registries are set up for administrative purposes to record patients with individual diagnoses and usually do not include detailed information about individual patients or their disease course. Some contain information on medications, hospitalizations, and healthcare use. Moreover, the validity of the diagnosis is often unproven. Registries for clinical trials record patients with the disease in question and include only the minimum information necessary to determine whether a patient is eligible for a clinical trial. Registries for genetic studies include patients with a particular disease (and usually also healthy controls) and minimal disease process information, but detailed genetic analyses information. Registries for biologics usually include only the minimum information necessary to determine therapy response and any particularly adverse events that relate to the therapy. Registries for longitudinal observational studies usually include more detailed information and are generally considered databases as opposed to registries (Table 1). Compared to clinical trials, longitudinal databases include all patients, record all drugs, provide longterm observation with a large sample, have inclusive information, and record all possible outcomes. Thus, databases allow the prospective collection of data from a large number of patients and use standardized protocols, including clinical, laboratory, imaging, and genetic data. These data are collected over a long observation period, which allows for varied presentations and courses to be analyzed. In addition, databases require computer tracking of all information. This allows for the description of disease course and longterm medication complications, the understanding of pathogenesis, and the study of associations between disease course and drug therapy. In addition, this provides insight into disease progression and allows researchers to plan for future trials.

To gain the most benefit from databases and to be able to

Personal non-commercial use only. The Journal of Rheumatology Copyright @ 2018 . All rights reserved. 
Table 1. Information collected in different registry types.

\begin{tabular}{ll}
\hline Type of Registry & Information Collected \\
\hline $\begin{array}{l}\text { Administrative } \\
\text { Clinical trials }\end{array}$ & $\begin{array}{l}\text { Demographic } \\
\text { Demographic plus some clinical information, } \\
\text { especially drugs } \\
\text { Proband and family information including } \\
\text { enough details to define a phenotype, together } \\
\text { wenetic studies }\end{array}$ \\
$\begin{array}{l}\text { Demographic, clinical, and therapeutic infor- } \\
\text { mation to determine response and adverse events } \\
\text { As much detail as possible to follow disease } \\
\text { progression and identify new features }\end{array}$
\end{tabular}

replicate or increase the power of specific observations, it is important that similar registries have the same information collected in a similar way. Thus, clinical and laboratory assessment should be confirmed to be similar and the consistency of variables must be assured. The actual platform need not be the same as long as harmonization between items is confirmed. Whatever database platform is used, it must allow for the easy transfer of data to a statistical system for data analysis.

It is important to avoid selection bias and demonstrate internal and external validity. Methods of observation and measurement must be clearly defined, and complete followup should be attempted to avoid information bias. It is also important to consider confounding factors such as time and intervention, although these may be overcome by design and analysis.

Dr. Gladman provided an example of the database from the University of Toronto Psoriatic Arthritis Program. The Toronto cohort currently includes 1450 patients and has been operating since 1978. Patients are assessed at 6- to 12-month intervals according to a standard protocol ${ }^{2}$. The reliability of joint assessment has been proven through a number of studies $^{3,4}$, and the radiographic method has also been proven to be reliable ${ }^{5}$. In addition, patients lost to followup and those followed regularly had similar disease characteristics at presentation $^{6}$.

Dr. Gladman highlighted the challenges of setting up a computer database, including quality assurance, data entry costs, issues with exporting data for statistical analysis, and the large number of staff required to maintain a database. Even with these challenges, the database provided the substrate for many investigations.

\section{Current and Proposed PsA Cohorts}

Dr. Marijn Vis (Rotterdam, the Netherlands) discussed the Dutch cohort of PsA, which is composed of 40 rheumatologists from 11 hospitals in the southwest of the Netherlands. The Dutch cohort's mission is to improve care for patients with PsA through education, research, and standardization. To set up their database, the Dutch cohort involved rheuma- tologists and patients with unmet needs who set up a clinical and science committee. Members contributing to the cohort own their own data. If possible, laboratory results, medication, and other data are taken directly from the hospital data warehouse, with patient questionnaires completed online. These data are all imported into 1 database. Data warehouses are used to store data from the hospitals' electronic patient files, and data collection is currently semiautomatic. To date, there are over 500 patients included in this database. In addition to the scientific use of data, the data will also be used, together with the automatic data import, to create a support tool for clinical care. The aim is to develop a decision-support system to assist physicians in using the appropriate treatment for the right patient based on the information collected in the database.

Drs. Laura C. Coates (Oxford, UK), William Tillett (Bath, UK), and Deepak R. Jadon (Cambridge, UK) presented a collaboration from the United Kingdom that will establish a cohort with embedded trials using a new methodology. This cohort will recruit patients from Oxford, Bath, and Cambridge starting in 2018. The Trials within Cohorts (TWiCs) or cohort multiple randomized controlled trial design will be used and was first published in 2010. This method recruits a central cohort having "treatment as usual" with regular observations and then adds pragmatic trials of alternative therapies. Eligible patients for trials are identified in the cohort and randomized to the offer of an intervention or to remain as controls in the cohort.

This design is particularly useful for open-label efficiency comparisons of therapeutic interventions with "treatment as usual" as the comparator. It is ideal for chronic conditions and where expensive desirable treatments are being tested. It allows robust generalizability from studies to routine healthcare, avoids attrition and disappointment bias from controls in open-label studies because patients only receive information relevant to their care, aids recruitment to trials, allows routine collection of longterm outcomes, and increases efficiency with multiple trials within 1 cohort ${ }^{7}$.

In collaboration, members of the group are establishing a cohort of patients with early PsA who will all receive step-up treatment guided by a treat-to-target (T2T) approach. This will form the central cohort and will collect outcome data on a real-world feasible T2T model. At present, 2 interventional trials are planned: the first in mild PsA with low disease effect in which patients will not be prescribed the usual disease-modifying therapy, and the second in moderate-severe PsA in which patients will be offered more aggressive therapy.

A number of TWiCs studies are currently running across Europe with the majority in the oncology field. The UK cohort study will be the first in rheumatology and also one of the first TWiCs studies testing investigational medicinal products. This has required appropriate liaison with regulators during protocol development. There are chal-

Personal non-commercial use only. The Journal of Rheumatology Copyright @ 2018 . All rights reserved. 
lenges, including ethical and good clinical practice issues with consent, and that data collection must balance the robustness necessary for clinical trials and feasibility for regular clinic visits. Many people are unfamiliar with the TWiCs concept, which has made it important to connect and educate university staff, charity and industry funders, clinical trials units, and ethics and medicines regulatory authorities.

In Oxford, the cohort will be new and has been established specifically as a TWiCs study. In Cambridge, the cohort is new. In Bath, the cohort is preexisting. Discussions among the 3 centers have aligned outcomes and timepoints for data collection to allow this collaboration.

Dr. Jadon is harmonizing a group of 750 PsA cases, historically looked after by 10 consultants at the Addenbrooke's Hospital, into a single cohort looked after in a dedicated PsA service that started in 2015. Using the EPIC platform, clinical data are collected to an electronic patient record system. In late 2017, electronic tablets will be used to collect patient-reported outcome measures (PROM) in the clinic waiting area and from home. The cohort includes both inception patients and prospective, established PsA patients. The program includes a consultant, research fellow, resident, research nurse, and PsA specialist nurse. The program takes direct referrals from general practitioners, dermatologists, gastroenterologists, ophthalmologists, and internal referrals. All patients undergo a series of PROM according to a protocol, examination indices, imaging, and laboratory tests. Patients have the opportunity for education and counseling about their condition, as well as management by the PsA specialist nurse and doctors. They also have the opportunity to attend 6 monthly patient and family education evenings hosted by Dr. Jadon.

In keeping with GRAPPA recommendations for the management of $\mathrm{PsA}^{8}$, multispecialty working has been a tenet of providing a holistic PsA service. In 2016, a monthly dermatology-PsA multidisciplinary team (MDT) meeting, 2 monthly inflammatory bowel disease (IBD)-spondyloarthritis (SpA) MDT meetings, and 2 monthly hepatology-PsA MDT meetings were established. These MDT meetings are attended by consultants, trainees, fellows, and specialist nurses. Complex patients, diagnostic conundrums, and treatment escalation are discussed, with a view to ensuring more harmonized care of the many facets of psoriatic disease. The MDT have also forged screening initiatives for PsA in patients with psoriasis and for SpA in patients with IBD. The challenges of setting up this PsA service have included optimizing patient flow between the MDT, information technology that enables direct referrals to the PsA clinic, funding and implementing the electronic data collection initiative using tablets, funding dedicated PsA staff and job planning, and convincing commissioners and funders of the clinical and economic virtues such a service provides to both patients and the hospital.

Dr. Tillett (Bath, UK) presented a historical perspective on the Bath PsA cohort and the opportunities and challenges of integrating a new TWiCs cohort into an established cohort database. The Bath PsA cohort was set up in 1989 by Professor Neil McHugh to answer questions about the PsA disease pathogenesis, clinical manifestations, prognostic indicators, the natural course of disease, and the real-world effect of treatment. The cohort is a secondary-care cohort primarily serving the local community (95\%) with $5 \%$ of participants coming as tertiary referrals from farther afield in the United Kingdom. Therefore, the cohort broadly represents patients with PsA in the United Kingdom. Patients are recruited to the cohort with any disease duration, including both new and established diagnoses (thus, it is not purely an inception cohort). In addition to a baseline set of data, clinical, patient-reported, and radiographic data are collected at routine clinical reviews (every 3 mos for patients with active disease and every 6 mos for those with more stable disease). Additional cross-sectional and longitudinal substudies have been undertaken to answer specific questions over the last 28 years. Patients and clinicians have historically collected data on paper, and these data are then scanned into a database where they are monitored and validated by a database team.

Ensuring that the cohort fulfills the most up-to-date PsA classification criteria has been an important consideration over time. Initially, entry to the cohort was based on physician diagnosis, then the application of Moll and Wright criteria, and finally retrospectively applying ClASsification for Psoriatic ARthritis (CASPAR) criteria ${ }^{9}$. Several milestones have necessitated changes to the data collected such as the introduction of biologic therapies, the need for more clinical and mediation data collection and development, and changes to the Outcome Measures in Rheumatology (OMERACT) core set of domains as measures in randomized controlled studies and longitudinal observational studies ${ }^{10,11}$.

The decision to set up a new TWiCs subcohort in Bath coincided with a recognition of the importance of moving to electronic data collection and integration with other clinical systems to widen the routinely collected research data. It became clear after discussions involving the Oxford, Cambridge, and Bath cohorts that having a single data collection platform across sites would not be achievable with the different organizations' funding priorities and existing contract commitments. The solution was to avoid real-time data upload and instead upload study data from existing systems at set timepoints during the study. A decision was made to harmonize datasets based on the OMERACT core set using the best-validated and most feasible measures. Feasibility of data collection was a significant consideration because each site has different clinic structures and staff resources. A dataset that could be achieved at each site was negotiated.

Prior to the open discussion about cohorts, Dr. Philip J. Mease (Seattle, Washington, USA) discussed the Corrona

Personal non-commercial use only. The Journal of Rheumatology Copyright $\subset$ 2018. All rights reserved. 
registry, a consortium of over 100 investigative centers in the United States that began as a registry for rheumatoid arthritis (RA) and PsA, but more recently added patients with $\mathrm{SpA}^{12}$. The RA registry currently tracks over 44,000 patients. The PsA/SpA registry has enrolled more than 2500 patients and collects detailed information on clinical disease manifestations, including enthesitis, dactylitis, spine and skin disease, comorbidities, and treatment efficacy and safety. This registry identifies only imaging data as done in practice, and for PsA/SpA has no biobanking component at this time. Examples of publications from this registry are referenced $^{13,14,15}$.

During the discussion, GRAPPA members were interested in the difficulties of setting up the cohorts, the financial considerations, as well as the feasibility of collecting detailed information on all patients.

GRAPPA members have expressed interest in developing a GRAPPA database, a concept that was further discussed during the research meeting that followed the 2017 GRAPPA annual meeting.

\section{ACKNOWLEDGMENT}

The authors acknowledge the patients who have contributed their data to the respective cohort and database studies, medical and allied healthcare colleagues who assist in the collection and analysis of data, and administrators who ensure the smooth running of these endeavors.

\section{REFERENCES}

1. Gladman DD, Farewell VT. Longitudinal cohort studies. J Rheumatol Suppl. 2005 Jan;72:30-2.

2. Gladman DD, Shuckett R, Russell ML, Thorne JC, Schachter RK. Psoriatic arthritis (PSA) - an analysis of 220 patients. Q J Med 1987;62:127-41.

3. Gladman DD, Farewell V, Buskila D, Goodman R, Hamilton L, Langevitz P, et al. Reliability of measurements of active and damaged joints in psoriatic arthritis. J Rheumatol 1990;17:62-4.

4. Gladman DD, Cook RJ, Schentag C, Feletar M, Inman RI, Hitchon $\mathrm{C}$, et al. The clinical assessment of patients with psoriatic arthritis: results of a reliability study of the spondyloarthritis research consortium of Canada. J Rheumatol 2004;31:1126-31.
5. Rahman P, Gladman DD, Cook RJ, Zhou Y, Young G, Salonen D. Radiological assessment in psoriatic arthritis. Br J Rheumatol 1998;37:760-5.

6. Brubacher B, Gladman DD, Buskila D, Langevitz P, Farewell VT. Followup in psoriatic arthritis: relationship to disease characteristics. J Rheumatol 1992;19:917-20.

7. Relton C, Torgerson D, O'Cathain A, Nicholl J. Rethinking pragmatic randomised controlled trials: introducing the "cohort multiple randomised controlled trial" design. BMJ 2010;340:c1066.

8. Coates LC, Kavanaugh A, Mease PJ, Soriano ER, Laura Acosta-Felquer M, Armstrong AW, et al. Group for Research and Assessment of Psoriasis and Psoriatic Arthritis 2015 treatment recommendations for psoriatic arthritis. Arthritis Rheumatol 2016;68:1060-71.

9. Tillett W, Costa L, Jadon D, Wallis D, Cavill C, McHugh J, et al. The ClASsification for Psoriatic ARthritis (CASPAR) criteria-a retrospective feasibility, sensitivity, and specificity study. J Rheumatol 2012;39:154-6.

10. Gladman DD, Mease PJ, Strand V, Healy P, Helliwell PS, Fitzgerald $\mathrm{O}$, et al. Consensus on a core set of domains for psoriatic arthritis. J Rheumatol 2007;34:1167-70.

11. Orbai AM, de Wit M, Mease P, Shea JA, Gossec L, Leung YY, et al. International patient and physician consensus on a psoriatic arthritis core outcome set for clinical trials. Ann Rheum Dis 2017;76:673-80.

12. Kremer JM. The Corrona US registry of rheumatic and autoimmune diseases. Clin Exp Rheumatol 2016;34 Suppl 101:S96-9.

13. Mease PJ, Karki C, Palmer JB, Etzel CJ, Kavanaugh A, Ritchlin CT, et al. Clinical characteristics, disease activity, and patient-reported outcomes in psoriatic arthritis patients with dactylitis or enthesitis: results from the Corrona Psoriatic Arthritis/Spondyloarthritis Registry. Arthritis Care Res 2017;69:1692-9.

14. Mease PJ, Karki C, Palmer JB, Etzel CJ, Kavanaugh A, Ritchlin CT, et al. Clinical and patient-reported outcomes in patients with psoriatic arthritis (PsA) by body surface area affected by psoriasis: results from the Corrona PsA/Spondyloarthritis Registry. J Rheumatol 2017;44:1151-8.

15. Harrold LR, Stolshek BS, Rebello S, Collier DH, Mutebi A, Wade $\mathrm{SW}$, et al. Impact of prior biologic use on persistence of treatment in patients with psoriatic arthritis enrolled in the US Corrona registry. Clin Rheumatol 2017;36:895-901. 